\title{
La mémoire retrouvée : le centenaire du CCF et la recherche de son histoire
}

\section{Michel Germain}

\section{(2) OpenEdition}

12 Journals

Édition électronique

URL : http://journals.openedition.org/communicationorganisation/1827

DOI : 10.4000/communicationorganisation. 1827

ISSN : $1775-3546$

Éditeur

Presses universitaires de Bordeaux

Édition imprimée

Date de publication : 1 novembre 1995

ISSN : 1168-5549

Référence électronique

Michel Germain, "La mémoire retrouvée : le centenaire du CCF et la recherche de son histoire », Communication et organisation [En ligne], 8| 1995, mis en ligne le 26 mars 2012, consulté le 19 avril 2019. URL : http://journals.openedition.org/communicationorganisation/1827 ; DOI : 10.4000/ communicationorganisation. 1827

Ce document a été généré automatiquement le 19 avril 2019

(c) Presses universitaires de Bordeaux 


\title{
La mémoire retrouvée : le centenaire du CCF et la recherche de son histoire
}

\author{
Michel Germain
}

1 «Il y a deux Histoires : l'Histoire officielle, menteuse qu'on enseigne, l'Histoire ad usum Delphini; puis l'Histoire secrète, où sont les véritables causes des événements" soulignait Honoré de Balzac $^{1}$. Peut-être doit-on à une recherche de sens très contemporaine le réel intérêt aujourd'hui porté à l'histoire des entreprises. Elle s'affirme à une époque d'intenses mutations marquées, pour les activités industrielles comme de service, par la nécessité accrue du changement et de l'adaptation à un environnement en constante évolution. La sauvegarde de la mémoire - en voie de disparition - de nombreuses entreprises constitue à l'évidence un enjeu d'envergure pour notre siècle finissant. Ce champ à part entière de l'histoire ne bénéficie pas toujours d'un niveau de conscience ou d'une attention suffisante. Les dirigeants d'entreprises, il est vrai, portent un intérêt plus manifeste aux enjeux économique et la rentabilité immédiate de l'activité dont ils ont la responsabilité, plutôt qu'à sa réalité socioculturelle.

2 Pour beaucoup, l'histoire d'entreprise appartient cependant aux préoccupations majeures de la fin du XXe siècle. Alain-Charles Martinet ne manque d'ailleurs pas de s'interroger sur ce «Besoin curieux (celui de l'histoire) à une époque où il n'est question que de change ment, de "nouvelles technologies», de futur ${ }^{2}$. D'une part, le monde de l'industrie et l'univers des activités de services poursuivent leurs mutations sur un rythme accéléré. Ils s'éloignent chaque jour davantage de leur état originel. D'autre part, l'économie se trouve pressée par d'incessantes évolutions. Elle en vient à négliger les traces éparses, les signes - encore présents mais fragiles - de la mémoire de ses techniques, de ses entreprises, de ses industries. À l'instar de Joseph Schumpeter ${ }^{3}$, nombre de responsables d'entreprise ont en effet compris qu'" Il serait illusoire de croire que l'on comprendra quoi que ce soit aux phénomènes économiques sans maitriser suffisamment les données historiques ». 


\section{Quelques constats préliminaires}

3 Sans volonté d'exhaustivité, plusieurs observations peuvent être faites sur la situation actuelle de l'histoire d'entreprise.

\section{$1^{\text {er }}$ constat}

L'entreprise n'accorde pas à son histoire une attention suffisante.

5 Confrontée aux impératifs du changement, elle fonde sa stratégie sur une vision d'ellemême plus projective que rétroactive.

\section{$2^{\mathrm{e}}$ constat}

L'histoire des entreprises appartient aussi à la collectivité nationale.

7 Elle constitue sans aucun doute un bien propre à l'entreprise et compte au nombre de ses actifs. Cependant, l'entreprise citoyenne, insérée dans le cadre social et culturel de la nation, se trouve confrontée au devoir moral de sauvegarde de sa propre mémoire.

\section{$3^{\mathrm{e}}$ constat}

8 Des pans entiers de la mémoire des entreprises disparaissent. Le rôle croissant joué par les technologies liées à l'informatique entraîne la destruction, souvent abusive, d'archives considérées comme obsolètes et encombrantes. Un plan d'urgence devient de ce fait impératif.

\section{$4^{\mathrm{e}}$ constat}

La mémoire des hommes disparait avec leur départ de l'entreprise.

10 Les changements sociologiques du travail entrainent le départ anticipé, en grand nombre, de salariés expérimentés. La collecte des témoignages de ces détenteurs de la mémoire collective présente un intérêt évident pour l'entreprise et la collectivité nationale.

\section{$5^{\mathrm{e}}$ constat}

11 Les entreprises sont à la recherche de sens.

12 La stratégie réactive de l'entreprise contemporaine, confrontée à un besoin de signifiance, nécessite la formalisation d'un ancrage destiné à lui permettre d'échapper à l'instant, d'inscrire son action dans la durée.

\section{$6^{\mathrm{e}}$ constat}

13 L'entreprise est confrontée au syndrome de l'éphémère. La réduction progressive de la durée moyenne de l'expérience de travail de chacun, au sein d'une entreprise définie, du fait de la pluralité nécessaire d'expériences différentes dans une même carrière, nécessite une explicitation plus nette de la communauté et des règles qui la régissent. 


\section{$7^{\mathrm{e}}$ constat} pèse sur elle en raison des mutations fréquentes qu'elle vit, des modifications de ses organisations, de la mobilité des hommes, de la réduction sensible du cycle de vie des projets mais aussi de l'augmentation considérable du nombre de ces derniers (ainsi ces éphémères "projets-kleenex »). Capitaliser le savoir-faire, gérer les compétences trouvent un regain d'intérêt en raison des perspectives qu'ouvrent les nouvelles technologies de l'information et notamment la gestion électronique de documents. L'entreprise commence à explorer ces pistes prometteuses qui vont lui permettre, à brève échéance désormais, de procéder à la modélisation systémique de l'ensemble de ses connaissances, de créer une architecture des systèmes d'information débouchant sur des outils d'aide au diagnostic et à la décision. De nouveaux environnements apparaissent. Ils permettent déjà -tel Nemesis TK de Cisi Ingénierie - de communiquer en langage naturel avec une base de donnée couvrant des champs d'expérience très divers et d'exploiter sur le mode d'association "hypertexte "les différents domaines d'information offerts. L'accès au savoir se trouve considérablement facilité dès lors qu'une cartographie des connaissances dresse en premier lieu un inventaire des différents domaines de pensée pour les relier de façon interactive. L'état des connaissances permet alors une analyse des forces et des faiblesses, des menaces et des opportunités. Il permet d'aboutir en certains cas à une analyse de risque et à l'établissement de scénarios prospectifs.

\section{Quelles réflexions ont incité le CCF à s'intéresser à son histoire}

De façon indéniable, la perspective de la célébration du centenaire du CCF, en 1994, a constitué le processus déclencheur d'une démarche historique inscrite dans la durée. Un temps relativement réduit, compte tenu de l'ampleur du sujet, a séparé le début des investigations, en 1989, de leur concrétisation cinq ans plus tard sous les différentes formes que présente cet article. Cette limite temporelle a constitué tout à la fois une gêne et un puissant stimulant. Une gêne en ce sens qu'elle n'a pas permis une investigation aussi large et aussi approfondie qu'il était possible de le souhaiter pour des raisons pratiques de temps, de moyens et de disponibilité. Un stimulant car l'échéance fixée obligeait à reconstituer, en l'espace des cinq ans impartis au chantier, ce que l'entreprise n'avait réalisé en 95 années d'activité. Enfin, il importe de préciser qu'avec clairvoyance Charles de Croisset, le Président du CCF en exercice, a su donner au centenaire l'importance symbolique qu'il pouvait revêtir. D'une part il s'est investi de façon personnelle dans les manifestations, d'autre part il a accordé aux opérations de communication, tant internes qu'externes, les moyens qu'elles nécessitaient. 
17 Parmi les différentes intentions qui ont présidé à la démarche historique, il est possible de citer :

18 - une recherche d'explicitation de l'identité et de la culture de l'entreprise. Le CCF se définit dans l'univers bancaire comme une entreprise à taille humaine (6 500 salariés), marquée par une forte empreinte de tradition et de modernité. Cette tradition tient à l'ancienneté de son activité et à l'accompagnement dans la durée de clients centenaires. Cette modernité résulte d'une volonté permanente de la banque d'innover. À titre d'exemple, dans un passé proche le CCF s'est engagé dès 1983 dans le domaine de la télématique bancaire en proposant à ses clients Vidéobanque. De façon plus récente, en mars 1995, il a été le premier établissement bancaire agréé par le gouvernement dans le cadre de l'appel d'offres sur les autoroutes de l'information. L'investigation entreprise a permis de mieux comprendre, pour reprendre l'expression de Jean-Paul Sartre, que " Nous vivons dans l'histoire comme un poisson dans l'eau, nous avons une conscience aiguë de notre responsabilité historique $»^{5}$

19 - une recherche de sens. Dans un univers professionnel fortement concurrentiel, la recherche historique réalisée a eu pour premier but de contribuer à la formalisation de ce " profil de distinction «qu'évoque P. Bourdieu, nécessaire à toute entreprise pour échapper à la banalisation. Elle a aussi participé à la compréhension et à l'appropriation, par le personnel, des caractéristiques propres de la personnalité de la banque. En complément des caractéristiques propres liées aux services et aux produits proposés, l'histoire permet d'accroitre la différenciation. Elle offre, en outre, la compréhension, pour reprendre l'expression de Cournot, de "L'étiologie historique, analyse des causes ou des enchainements de causes qui ont concouru à amener les événements dont l'histoire nous offre le tableau " ${ }^{6}$

20 - la restauration de la mémoire collective. Des fractures de culture ont été constatées en plusieurs occasions dans l'histoire de l'entreprise. En certains cas, elles trouvent leurs origines dans les différences de style managér de certains présidents dont la personnalité a fortement marqué l'entreprise. En d'autres cas, elles tiennent aux événements fortement connotés survenus dans l'existence du CCF, ainsi la nationalisation puis la privatisation de l'entreprise. Ces facteurs ont nui à la perception claire, dans la durée d'une certaine perspective structurée. L'histoire établit un lien entre ces événements et restitue leur importance respective et relative.

21 - la compréhension de l'organisation. L'évolution de l'entreprise à travers le siècle s'est accompagnée d'une professionnalisation progressive des métiers qu'elle exerce. Au métier originel unique et à la polyvalence première des fonctions s'est substitué, principalement depuis le début des années 80 , un corporatisme croissant. L'une des raisons de cet état de fait tient à la spécialisation des métiers, aux développements des techniques qui leur sont liées, mais aussi à la mondialisation de l'activité bancaire et financière. Le développement de niches de compétences très particulières s'est affirmé. Dans ce contexte, l'histoire contribue à une meilleure compréhension d'un univers de complexité.

22 - le renforcement de l'appartenance et de l'appropriation. Dans un contexte économique global marqué par un nécessaire raccourcissement de la stratégie, pour des raisons de réactivité et d'adaptation aux besoins du marché, la nécessité d'un renforcement du sens de la communauté s'affirme. Cette dernière ne s'affirme pas tant par les produits réalisés que par les comportements et attitudes que pratique la 
collectivité dans son ensemble. Comprendre ce que l'on peut dire et ce que l'on peut faire permet de « décoder » les régies tacites d'une communauté. formes mêmes des actions destinées à célébrer le centenaire, ce travail indispensable visait à effectuer un recensement préliminaire de l'étendue du corpus historique et des matériaux disponibles dans les archives administratives de la banque. Cette étape initiale de l'inventaire fut confiée à Jean-Pierre Daviet ${ }^{7}$. Professeur des universités, spécialiste de l'histoire des entreprises, ce dernier anime un séminaire d'« Histoire de la gestion des entreprises » à Paris I ainsi qu'un cours à l'Ecole normale supérieure de Cachan intitulé « Entreprise et histoire, $\mathrm{XIX}^{\mathrm{e}}$ et $\mathrm{XX}^{\mathrm{e}}$ siècle « A partir des rapports du conseil du CCF, des principaux documents administratifs (comités de direction, conseils d'administration, etc), il proposa un premier texte.

Ce travail, d'excellente facture, suscita un premier débat dans l'entreprise. Il souleva notamment la nécessité d'approfondir certains points plus obscurs de l'histoire de la banque et d'entreprendre une recherche complémentaire. La sécheresse administrative du ton des rapports ne livre pas, comme chacun sait, le cheminement des réflexions qui mène aux décisions. Elle ne restitue ni les enchaînements multiples, ni les arbitrages qui aboutissent à une prise de position. Est-il besoin de préciser ici que la tradition de discrétion, de mise dans l'univers de la banque, conduit le plus souvent, dans la rédaction des textes, a une pondération des propos. Pour cette raison, il sembla rapidement nécessaire d'élargir la collecte d'information dans une direction complémentaire, car pour reprendre l'expression de Sainte-Beuve "Il y a une sorte d'histoire qui se fonde sur les pièces mêmes (...). Il y a une histoire d'une toute autre physionomie (...) écrite par des acteurs et des témoins ${ }^{8}$. Dans cet esprit, une première piste fut celle consistant à interroger des salariés en activité, en privilégiant ceux qui, proches de la retraite, effectuèrent la totalité de leur carrière dans la banque. Une seconde voie fut celle de la consultation des retraites. Deux mille d'entre eux adhèrent aujourd'hui encore, en effet, à l'Amicale des retraites. Les membres de cette association n'ont, pour la plupart, connu tout au long de leur carrière d'autre entreprise que le CCF. Les souvenirs de certains d'entre eux, retirés depuis longtemps mais toujours en vie, couvrent près d'un demi-siècle de la vie de la banque. Plus encore, d'autres sont, par une tradition en vigueur jusque dans les années 70 , entrés dans l'entreprise sur la recommandation d'un proche parent qui y avait lui même effectué auparavant sa propre carrière. Cette forme de recrutement s'est considérablement réduite et a aujourd'hui quasi disparue. N'oublions pas, à cet égard, que le fondateur du CCF, 
Benjamin Rossier, avait choisi de créer une banque à taille humaine, proche de ses clients. Suisse d'origine, homme de principe et d'une grande moralité, il accorda dès la fondation de la banque une grande importance à la qualité des hommes.

Pour recueillir l'information que pouvaient apporter les actifs et les retraites une grille méthodologique d'interview fut constituée. Son but était de favoriser le traitement de l'information rassemblée. La possibilité d'un tri efficace permit en effet d'établir une comparaison entre les différentes données collectées. Les renseignements ainsi accumulés permirent de compléter nombre de zones d'ombres constatées. Une chronologie de plus en plus détaillée et fournie, au fur et à mesure de l'avancement du travail historique, constitua l'indispensable fil rouge permettant de classer les informations. Deux champs de classification de documents furent établis en parallèle. L'un concernait les informations disponibles sous forme de textes, l'autre celles qui l'étaient sous une forme iconographique. Il est révélateur d'observer, à ce dernier sujet, qu'a la petite vingtaine de documents photographiques d'époque, disponibles en 1990, se substitua une moisson croissante. Fournie pour une part par les actifs et les retraites, elle a porté aujourd'hui le nombre d'illustrations (gravures et photos d'époque sur les bâtiments, les hommes au travail, le métier bancaire) à prés de six cents documents qui sont la propriété du CCF. Cette recherche fut bien sur complétée par la collecte des objets révélateurs de l'évolution du métier bancaire. Au total, une cinquantaine d'objets sont aujourd'hui rassemblés dans un local affecté depuis deux ans, au siège des ChampsElysées, à la conservation du fonds historique. Parmi ces témoins d'une époque révolue figurent notamment les différentes machines qui ont permis, depuis un siècle, l'évolution des techniques de calcul.

De façon parallèle, un autre travail fut mis en chantier, de façon interne. Il consista à rassembler l'information qui pouvait encore être retrouvée sur les différentes banques rachetées par le CCF au cours de son histoire. Ces établissements, d'implantation régionale dans l'ensemble, ont disparu depuis longtemps, en raison de leur fusion avec la banque. Ils ont toutefois laissé des traces dans l'histoire locale. Tel est le cas de la Banque de Mulhouse, née en 1871 et rachetée par le CCF en 1919, ou de la Banque de Bordeaux, acquise en 1919.

\section{De la collecte de l'information historique à la démarche communication}

En parallèle de la démarche d'investigation quatre étapes peuvent être distingués dans la démarche de communication. Elles visaient la progressive sensibilisation des publics internes et externes du CCF à la révélation de l'histoire.

31 En 1989 et 1990, la première étape fut celle de la recherche des textes fondateurs et de la consolidation des données qui ne donna lieu, au sens propre, à aucune communication interne. Un principe de prudence fut en effet observé. Il consistait à ne pas faire état des travaux en cours tant qu'une maitrise suffisamment complète et approfondie du dossier ne serait pas acquise. Pendant cette période, un recensement précis des personnes, en activité ou en retraite, susceptibles d'être abordées dans un second temps fut toutefois dressé.

2 En 1991 et 1992, la seconde étape consista en une sensibilisation du personnel et des retraites à l'histoire de la banque. À titre d'exemple, pendant deux années un encart 
historique intitulé «Futur Antérieur «mit l'accent, dans le magazine interne diffusé à l'ensemble du personnel en activité et aux membres de l'amicale des retraites, sur les pages les plus significatives de la chronique du $\mathrm{CCF}^{9}$. Cette démarche d'information interne fut complétée par des animations destinées à provoquer un retour d'information. Dans le cadre d'un concours photo interne, l'accent fut ainsi mis, en 1993, sur le patrimoine du CCF.

En 1993, la troisième étape fut celle de la mise en place du plan de communication prévu pour la célébration du centenaire en 1994. À ce moment furent déterminées les différentes actions de communication destinées aux publics internes et externes. Ce plan de communication prévoyait, en premier lieu, la forme des supports majeurs d'information destinés à sous-tendre les différentes opérations prévues. Ces outils sont au nombre de deux :

34 - en premier lieu figure la réalisation d'un livre intitulé 1894-1994, Une banque dans le siècle ${ }^{10}$. Destiné a un large public, il concrétise les enseignements livrés par la collecte d'informations. Il fut donc décidé, dans un but de vulgarisation, que cet ouvrage serait accessible et attrayant. Dans cette perspective, trois niveaux de lecture furent déterminés. Ils formalisent la construction graphique et la présentation des quatre parties du livre:

35 - une chronologie détaillée, en ouverture de chaque partie, précise pour chacune des années du siècle, en caractère gras, les événements importants survenus au CCF. En caractère maigre, les principaux événements qui ont marqué l'actualité nationale et internationale de l'année sont mentionnés à la suite des précédents,

36 - un texte fourni relate, ensuite, l'évolution de la banque. Il est complété, en annexe, par de nombreux encadrés. Il s'agit, suivant le cas, de commentaires consacrés à un personnage important de la banque ou à l'explicitation nécessaire d'un événement capital survenu à un moment précis de son histoire,

37 - une iconographie abondante et légendée - plus de 250 photos au total - forme le troisième niveau d'information. Ces documents illustrent l'évolution des métiers, mettent l'accent sur les techniques oubliés, rappellent le visage de ceux qui, un siècle durant, construisirent avec persévérance l'établissement bancaire d'aujourd'hui.

38 Au total, près de 20.000 de ces ouvrages furent diffusés en un peu moins d'un an auprès du personnel, des retraités, des clients du CCF. Favorablement accueilli par ces publics fort différents dans leurs attentes respectives, l'ouvrage constitue désormais le document de référence interne, ce livre de mémoire qui faisait défaut.

- en second lieu, un film historique fut produit. Destiné à servir d'introduction colorée et animée, pendant toute l'année 1994, aux actions de communication internes et externes de la banque, il fut réalisé pour le CCF par Didier Pourcel. À la veille des manifestations de célébration du centenaire du cinéma, le CCF - l'aîné d'un an de cette invention majeure s'est rappelé qu'au début du siècle il fut le banquier avisé de Charles Pathé mais aussi de Léon Gaumont. Pour cette raison, à l'heure du « tout vidéo «, le choix fut fait de réaliser un film en $35 \mathrm{~mm}$. Dans sa première moitié, ce document présente, dans le cadre d'une fiction interprétée par Jean Rochefort et Mariène Weber, les principaux événements intervenus dans la vie du CCF. De nombreuses scènes rétrospectives, en «flash back « et en noir et blanc, marquent de façon claire la séparation entre la fiction et la réalité. Elles illustrent l'histoire de l'entreprise en découvrant des photos réelles de personnages, d'activités et de lieux empruntées au fonds iconographique du CCF. Dans sa seconde 
moitié, ce même film, emprunt jusque-là d'un certain classicisme, bascule dans le XXI siècle et dans le domaine de l'image virtuelle. Cette transition manifeste la tradition d'innovation d'une banque, déjà pionnière au début du siècle quand elle soutint la démarche de Guglielmo Marconi, de Charles Pathé ou de Léon Gaumont parmi d'autres. Elle fut aussi novatrice quand elle finança, à Paris, la première ligne du métropolitain, quand elle soutint Pierre Azaria dans son projet de fondation de la Compagnie Générale d'Electricité, devenue depuis lors Alcatel-Alsthom, quand elle participa au développement de Nestlé. Au personnage réel de Jean Rochefort, dans la première partie du film, succède dans cette seconde partie sa représentation virtuelle née d'une modélisation en trois dimensions dans les laboratoires de l'université de Jussieu. Au préalable, un Cyberware a permis d'enregistrer sur ordinateur le visage et le corps du comédien pour en restituer les moindres expressions et les mouvements. Ce film a obtenu le prix de l'audiovisuel d'entreprise au Festival de Biarritz, en juin 1994. Atteignant son objectif de communication externe, il a été abondamment cité par la presse spécialisée et par la télévision notamment dans Culture Pub sur M6, Décryptage sur FR3 et Nulle part ailleurs sur Canal +.

En 1994, la quatrième et dernière étape fut la réalisation des actions prévues dans le plan de communication du centenaire. Echelonnées dans leur déroulement sur une année entière, elles étaient destinées, de façon alternative mais équilibrée, aux publics internes et aux publics externes.

41 Pour le public interne, le début de l'année 1994 fut marqué par la diffusion à chaque salarié d'une lettre du Président précisant l'enjeu de l'événement. Ce document était accompagné d'une brochure historique présentant la chronologie du CCF. Cette publication visait à révéler les grands traits de l'histoire avant que ne commencent les manifestations du centenaire. L'événement le plus marquant du centenaire fut un mois plus tard, le 3 février 1994, la "convention" à laquelle Charles de Croisset associa l'ensemble du personnel. Elle rassembla au même moment, dans dix villes de France reliées de façon interactive par satellite, personnel actif et retraité. Pour mémoire, neuf mille personnes furent conviées à la manifestation. Cette opération constitua une première dans l'histoire du CCF en raison de son ampleur et de l'association, dans un même cadre, des acteurs passés et présents de l'entreprise.

La convention fut en fait l'aboutissement d'un important travail de réflexion mené auprès des salariés en 1993 sur la culture du CCF. À l'automne, en effet, des groupes de travail furent constitués. Composés de représentants des différents métiers de la banque, ils se réunirent sous la conduite d'animateurs dans le but de définir leur propre vision de l'entreprise. Ils furent en même temps appelés à dégager, des enseignements livrés par l'histoire de l'entreprise, ce qui leur semblait constituer les traits caractéristiques de l'identité du CCF. Cette investigation conduisit notamment à la formalisation d'un "verbatim », vocabulaire commun définissant les qualificatifs exprimés par le personnel lorsqu'il définit l'établissement bancaire.

Pendant les deux heures de la manifestation se succédèrent, en totale interactivité sur les différents sites de la convention, des interventions et des témoignages variés appelés à illustrer la personnalité du CCF. Les trois temps de l'opération évoquèrent successivement le passé, le présent et l'avenir, dans un ordre diachronique tendant à exprimer l'idée que, si le passé éclaire le présent, c'est pour permettre une projection de ce dernier vers l'avenir. Voltaire n'écrivait-il pas, à ce sujet que «La mémoire nourrie et exercée est le meilleur support de l'imagination ». Les témoignages des acteurs de l'entreprise furent eux- 
mêmes équilibrés par ceux de clients de la banque, venus non pas tant expliquer les raisons pour lesquelles le CCF a accompagné leur développement depuis un siècle exemple des Galeries-Lafayette ou de la Compagnie Générale d'Electricité - que celles pour lesquelles il serait associé à leur propre avenir. Derrière cette explication transparaissait une évidente volonté de faire passer auprès du personnel du CCF un message de dynamisation ainsi que certains axes de progrès et de remise en cause. Il est vrai, pour reprendre l'expression de Fernand Braudel que «l'avenir ne se prévoit pas, il se prépare ».

44 Pour les publics externes, ceux constitués par les prescripteurs et les clients, les actions furent de différents ordres. En premier lieu, il fut décidé d'utiliser la vitrine naturelle et privilégiée constituée par le siège implanté au 103 de l'avenue des Champs-Elysées. Cet imposant bâtiment, site classé de plus de 70 mètres de façade, fut en effet édifié entre 1897 et 1899 par Georges Chedanne, Prix de Rome, pour le compte de George Nagelmackers, le directeur des Wagons-Lits. Ce dernier en fit l'Elysée Palace, l'un des hôtels les plus réputés de la capitale au début du siècle. Il fut la résidence de passage du Shah de Perse, du Maharadjah de Kapurthala ou du prince Youriewski. Racheté par le CCF à la fin de la première guerre mondiale, l'édifice abrite la direction générale de la banque depuis le mois de janvier 1922. En 1994, une brochure intitulée " Mémoire d'un lieu » fut d'ailleurs consacrée à une évocation historique de ce site d'exception. Ce document, destiné d'une part aux collaborateurs du CCF, entendait les sensibiliser à l'intérêt culturel d'un cadre étonnant dans lequel, par un caprice de l'histoire, Mata Hari vécut ses dernières heures de liberté, où le journaliste Léon Daudet et et le poète Paul-Jean Toulet avaient leurs habitudes avant la première guerre mondiale. Cette plaquette fut remise également aux clients accoutumés à se rendre à l'Agence Centrale, qui depuis soixantedouze ans, occupe le rez-de-chaussée du bâtiment, de plain-pied sur les Champs-Elysées.

Cette position sur la principale avenue de Paris, exceptionnelle dans le milieu bancaire concentré par habitude dans le quartier des grands boulevards, résulte d'une intuition clairvoyante de Benjamin Rossier. Au début du siècle, il estima l'un des premiers que l'expansion de la capitale s'effectuerait à l'ouest de Paris. Depuis cette époque, le CCF est connu pour cette raison sur les principales places étrangères comme "la banque des Champs-Elysées ". Il fut donc décidé de tirer parti de la façade du bâtiment en transformant celle-ci en diaporama géant, à l'occasion de la période particulièrement propice des illuminations de l'avenue, à l'époque de la nouvelle année. La façade fut donc entièrement éclairée et colorisée de façon à faire ressortir les moindres détails des sculptures. Certains des moulages de ces dernières, réalisés comme modèle par le sculpteur F. Sicard en 1898, dorment dans les réserves du Musée des Beaux-Arts de Tours. En outre, les cinquante principales fenêtres furent transformées en écran de projection sur lesquelles défilèrent 4000 images présentant les différentes expressions de la réalité actuelle et passée de l'entreprise. Dans le cadre de cette animation, les principaux clients parisiens furent invités au siège à l'occasion de cocktails. La projection du film Souvenirs à venir venait renforcer une présentation muséographique, objets bancaires, des affiches anciennes et des panneaux illustrés, l'histoire passée de la banque.

En second lieu, une série de manifestations furent organisées dans les principales régions d'implantation du CCF. Elles permirent de célébrer avec les clients de ces localités mais aussi avec les descendants des fondateurs (ainsi la présence de membres des families Aynard et Rossier à Lyon), le centenaire de la banque. En les associant à cet événement, il 
s'agissait aussi de manifester l'importance qu'attache à travers le temps la banque aux relations de confiance qu'elle noue avec ses partenaires.

En complément de ces opérations diverses, une information historique fut dispensée dans un certain nombre de publications. Le support institutionnel à large diffusion qu'est le rapport annuel du CCF présenta dans ses colonnes une chronologie de la banque, destinée à présenter à ses lecteurs -actionnaires, investisseurs institutionnels, prescripteurs l'histoire de l'entreprise. Ce document obtint d'ailleurs, à l'automne 1994, le prix du meilleur rapport annuel décerné par La Vie Française. Le magazine interne Ressources consacra pour sa part un numéro exceptionnel à l'ensemble des manifestations du centenaire et à une explication de l'ensemble de la démarche historique. À l'extérieur de l'entreprise, un encart historique fut diffusé au printemps 1994 dans le magazine Géo. Ce tiré à part fut également remis aux invités dans chaque opération régionale. Les manifestations du centenaire furent par ailleurs relayées dans la presse nationale et régionale. À titre anecdotique, Le Messager Suisse, la revue des communautés suisses de langue franchise, consacra un article au centenaire de la banque, en raison bien sur des origines helvétiques du fondateur de la banque.

\section{Poursuivre la démarche}

À l'évidence la célébration du centenaire du CCF a constitué un catalyseur auprès des salariés dans la prise de conscience de l'importance de l'histoire de leur entreprise. Ce type d'événement, plus qu'aucun autre, est sans doute nécessaire à la cohésion d'un groupe bancaire et financier. En règle générale, l'entreprise n'a que peu de temps en effet à consacrer au quotidien à son histoire en dépit de l'intérêt indiscutable que revêt une telle démarche. Si l'histoire donne un sens à l'action, si elle éclaire les modes de décision et les règles managériales de l'entreprise, elle est toutefois de peu d'usage dans l'exercice quotidien de la conduite d'une entreprise dès lors qu'il s'agit d'opérer des arbitrages immédiats sous la pression d'événements non récurrents. Elle est surtout, un vecteur de forte cohésion sociale en ce sens qu'elle offre un champ référentiel indiscutable, favorisant le dialogue et le partage.

Alors que la page se tourne, quelles conclusions dresser d'une année de communication d'exception centrée vers l'histoire? Pour l'heure se terminent les dernières actions destinées à sauvegarder pour l'avenir le travail de recherche réalisé. La numérisation du fonds iconographique constitué au cours des cinq dernières années est en cours. Un important travail de légende et d'indexation permettra de disposer, dans les prochains mois, d'une base informatisée permettant la recherche multicritère. Dans ce cadre, le «compact disc «servira désormais de support d'image. Il supprimera le support argentique, ses risques d'altération et permettra une protection parfaite et illimitée des documents. Le fonds d'affiches historiques détenu par le CCF - emprunts de la première guerre mondiale - a été complété par des acquisitions récentes. Les différents documents possédés - soit une cinquantaine d'affiches originales - ont fait l'objet de restaurations approfondies et d'entoilages par les établissements Quillet, spécialisés dans la réfection de documents. Les soins prodigués devraient assurer une sauvegarde pour les cinquante prochaines années. Le référencement de l'intégralité des objets - documentaires ou iconographiques - rassemblés dans le cadre des travaux de préparation du centenaire est destiné à contribuer, à une échéance ultérieure, à la constitution d'un fonds collectif dans le cadre d'un système d'information globalisé. 

perception du climat de communication et d'information, réalisé en décembre 1994 auprès d'un panel de 653 salariés, laisse apparaître des scores positifs. L'histoire a été plébiscitée pour sa capacité à faire mieux comprendre et connaitre le CCF ( 72 \% d'opinion positive). Elle a semblé bien exploitée en communication interne (73\%) et en communication externe (69\%). Le traitement de l'histoire dans le livre du centenaire a été apprécié par $82 \%$ des personnes. Le personnel estime avoir progressé de façon forte dans la compréhension qu'il a de son univers de travail. Il estime réussies et bien réalisées les manifestations auxquelles il a été convié. Ce satisfecit général rejaillit sur la perception de la communication de l'entreprise en général. Il témoigne sans doute du fait qu'un important travail de réflexion a été réalisé et qu'une réponse adaptée a été apportée à une attente jusque-là informulée de compréhension de la collectivité humaine.

En dépit de ces signes positifs, il convient d'éviter tout triomphalisme et de reconnaitre avec humilité les limites d'une telle démarche. L'histoire se révèle incapable de clarifier l'univers de la complexité auquel se trouve confrontée aujourd'hui une entreprise. Elle permet néanmoins de distinguer dans ce désordre apparent certaines lignes de forces, des convergences qui permettent de se livrer à des projections. À une époque où les impératifs de la conjoncture nécessitent des ajustements constants de cap, l'histoire permet de mieux saisir ce qui échappe à l'instant. En ce sens, une formalisation des attitudes liées aux us et coutumes propres à la profession s'affirme. Elle correspond, dans bon nombre d'entreprises, à l'expression de ce code de comportement, trop rarement concrétisé, que souhaitent dans leur grande majorité les salariés.

52 À la lumière de l'expérience citée en référence, sans doute peut-on considérer qu'un effort de recherche historique du type de celui mené au CCF peut être engagé sans grands moyens financiers et techniques (hormis ceux exceptionnels nécessités par la célébration du centenaire) dès lors que la volonté d'aboutir et la conviction sont réelles. Cela nécessite que les responsables en charge du projet soient dotés d'une marge d'autonomie et de décision suffisante. Sans doute y faut-il, aussi, une certaine part de passion pour trouver le temps et les moyens de mener à bien cette investigation quand il ne s'agit pas, pour la personne en charge de la mission, d'un plein temps incompatible le plus souvent avec les charges de travail d'une activité professionnelle. Dès lors que le temps se trouve compté et que l'échéance d'une célébration ne saurait à l'évidence être différée, une certaine angoisse naît de l'impérative nécessité d'être prêt à temps. Chaque jour apporte en effet sa part de découverte et d'approfondissement complémentaire.

En terme de collaboration, la solution adoptée par le CCF a consisté à allier des compétences extérieures solides - celles de Jean-Pierre Daviet - pour la première phase de la collecte historique, à celles d'un chef de projet interne au CCF - le responsable de la communication interne en l'occurrence - pour l'enrichissement de la collecte et le traitement de l'information. Cette formule semble la plus judicieuse en terme de rigueur. Elle permet en effet de conserver une nécessaire distance critique, d'éviter les débordements préjudiciables et de compenser les inévitables dérives que risquerait d'encourir un responsable unique confronté à sa propre subjectivité. Une telle démarche autorise en même temps l'accès à une diversité de sources procédant de la mémoire morte ou de la mémoire vivante. Qu'il me soit permis, ici, de rendre un sincère hommage à Jean-Pierre Daviet dont la probité, la rigueur, et la compétence historique ont constitué pour la banque un indéniable apport.

Communication et organisation, 8 | 2012 
54 En conclusion, une approche d'histoire d'entreprise ne saurait atteindre à sa véritable portée si elle ne s'insère dans une démarche de communication structurée et si elle ne repose sur des outils conformes aux objectifs qu'elle s'est fixée. Comme l'exprime AlainCharles Martinet, " La communication ne peut se passer de culture, laquelle ne signifie rien si elle n'organise pas la visibilité des choses et donc ne s'appuie pas sur l'histoire ${ }^{11}$. Dans la vie des entreprises, l'histoire reste condamnée a un rôle marginal à moins qu'elle ne soit associée à la stratégie de cette dernière et qu'elle s'exprime dans sa politique de communication. En ce cas, elle devient un "projet d'entreprise " à part entière. Elle participe à une démarche axiologique, au sens de «métaphysique du vouloir», par la capacité de mobilisation qu'elle suscite. Elle est en même temps cette démarche utilitaire qui participe à l'explicitation du présent et de l'avenir. Pour reprendre la formule d'Alain Baudant $^{12}$, «La mémoire fait de l'histoire ce moyen imparfait, parfois impur, qui permet de garder trace des fondements, des cheminements, des solidarités; qui peut favoriser l'osmose entre les compétences nouvelles et le savoir accumulé, faciliter les décisions s'il ne dicte pas des solutions. Après, inévitable, vient le pari ».

Le Crédit Commercial de France, groupe bancaire et financier dont le siège est à Paris, représente aujourd'hui plus de 10.000 personnes. Elles exercent leur activité dans 36 pays au monde et dans une cinquantaine de filiales. Fondée en 1894, la Banque Suisse et Française devint en 1917 le Crédit Commercial de France. L'absorption de plusieurs banques régionales (Banque du Colombier, Banque de Bordeaux, Maison Aynard \& Fils, Caisse de Crédit de Nice, Banque de Mulhouse, Banque de l'Artois) lui permit d'implanter en un demi-siècle un réseau qui comprend aujourd'hui 436 succursales et agences, dont 184 sous son enseigne propre. Son histoire fut notamment marquée par les personnalités fortes de Benjamin Rossier, son fondateur d'origine suisse, du Baron Davillier au milieu du siècle, de Jacques Merlin, dans les années 60. Plus récemment, la banque a été nationalisée en 1982, puis privatisée en 1987 lors de la présidence de Michel Pébereau auquel a succédé Charles de Croisset, l'actuel président, en 1993.

\section{NOTES}

1. BALZAC (de) Honoré. Les illusions perdues, t. IV, p. 1020.

2. V. MARTINET Alain-Charles. L'Histoire, un investissement production Revue Française de Gestion, $\mathrm{n}^{\circ} 70$, Paris, Septembre 1988

3. SCHUMPETER Joseph Aloïs. Capitalisme, socialisme et démocratie (1942), Payot, Paris, 1967.

4. VALERY Paul Variétés IV, 1938. p. 139.

5. SARTRE Jean-Paul. Situations II, pp. 40-41.

6. COURNOT Antoine Augustin. Traité de l'enchaînement des idées fondamentales dans les sciences et dans l'histoire. Chap. 1

7. DAVIET Jean-Pierre. Une multinationale à la française, Saint-Gobain -1665-1989, Fayard, 1989 Eurodif -1973/1993 Histoire de l'enrichissement de l'uranium, Fonds Mercator, 1993.

8. SAINTE-BEUVE Réflexion sur les lettres. Plon, Paris, 1941, p. 70.

9. ROSSIER Benjamin, correspondance adressée à Francisque AYNARD, 2 décembre 1916, archives du CCF. 
10. DAVIET Jean-Pierre et GERMAIN Michel. 1894-1994 Une banque dans le siècle Editions Textuel, Paris, 1994

11. MARTINET Alain-Charles. L'histoire, un investissement productif in Revue Française de Gestion. $\mathrm{n}^{\circ} 70$, Paris, Septembre 1988

12. BAUDANT Alain. De l'histoire à la stratégie d'entreprise : l'exemple de Saint-Gobain, Mémoire d'avenir, Edition Economica, Paris, 1987

\section{RÉSUMÉS}

Cet article aborde en deux temps une réflexion sur l'histoire d'entreprise. En premier lieu, il dresse un certain nombre de constats préliminaires sur l'intérêt porté aujourd'hui par les entreprises à leur histoire et sur la nécessité de la conservation de la mémoire. Quelques pistes sur les perspectives technologiques qu'ouvre la modélisation des connaissances sont par la même occasion esquissées. En second lieu, la démarche accomplie par le CCF à l'occasion de la célébration de son centenaire en 1994 est présentée de façon détaillée. L'article développe les intentions initiales, présente les méthodes d'investigation utilisées, définit le rôle des partenaires. Il évoque la démarche globale de communication réalisée par le CCF à l'intention de ses publics internes et externes (clients, prescripteurs). Par ailleurs, il présente les différents supports réalisés dans le cadre du centenaire (livre d'histoire, film, convention d'entreprise, opérations destinées à la clientèle, etc.).

Enfin, après une évocation des retombées du centenaire et de la poursuite de l'action de sauvegarde historique, une conclusion aborde la portée réelle d'une communication historique.

This article addresses the field of corporate history, in two parts. Its begins by reviewing the current way if interest among corporations for research into their past and the need to preserve historical records. It identifies a few areas in which new technologies based on modeling techniques offer promising prospects. Secondly. the author takes a defailed look at the program adopted by CCF at the time of the bank's centenary in 1994. The article examines the bank's initial objectives. describes how it went about achieving these, and defines the roles of the partners involved. Il discusses the overall Communications policy adopted by CCF for internal and external purposes (clients, decision-malers), and reviews different products developed to commemorate the centenary: a corporate history in book form. film, a company-wide agreement, special operations targeting clients, etc. After reviewing spin-offs of the centenary celebration and the banks on-going efforts to preserve records of its past, the conclusion considers the scope and impact of a Communications drive based on corporate history.

\section{AUTEUR}

\section{MICHEL GERMAIN}

Directeur de la communication interne du CCF (Head of internal Communications). Docteur es Lettres - Chargé d'enseignement au Celsa (Université Paris IV). 